\title{
A silver service at the front door of the hospital
}

\author{
Author: William Wareing
}

\section{Aims}

> Improve access to multidisciplinary team (MDT)-delivered, comprehensive geriatric assessment (CGA) to frail patients within the emergency department (ED).

$>$ Reduce inappropriate admissions of frail, older people to hospital.

> Reduce polypharmacy and inappropriate prescribing in older people within the ED.

> Improve links with and support to community services through the use of a 'silver service telephone'.

\section{Methods}

We developed an 'emergency geriatrics' MDT consisting of a consultant geriatrician, rapid response therapist, band 8 prescribing pharmacist and core medical trainee to work within our trust's ED from 9am-5pm, Monday to Friday. The consultant geriatrician also carried a mobile telephone to provide access to specialist advice to general practitioners and community practitioners regarding admission avoidance and frailty syndromes.

Service activity data was collected prospectively and validated by the trust's patient administration system

The service's activity and structure was developed using multiple Plan, Do, Study, Act (PDSA) cycles to improve productivity during the initial 3 months of operation

\section{Results}

Two-hundred and ninety-seven patients $(85 \pm 7.5$ years, $67 \%$ female with a clinical frailty score of $5 \pm 1.3$ ) were assessed by the team during the first 3 months. Of these, 152 (51\%) were discharged to the community, 27 (9\%) were directly transferred to an intermediate care facility, and $118(40 \%)$ were admitted to an acute hospital bed. Among the admitted patients, 39\% were treated by geriatricians, $36 \%$ by other medical specialties and $25 \%$ in an outlying ward.

An average of 0.86 medications ( 0.26 and 0.12 were in red and amber categories, respectively) were stopped per patient.

Forty-two percent of the 118 patients subsequently admitted to the hospital had been assessed as having no acute medical need for admission.
There was limited use of the silver service telephone despite several community care providers feeling that it would help to reduce ED attendances. This was found to be due to limited community knowledge of the service.

\section{Conclusion}

The 'front door geriatrics' model is effective in delivering CGA to frail patients within the ED and reducing inappropriate admissions to hospital. However, a further benefit could be achieved through prolonged out-of-hours service cover. The positive results demonstrated the need for a continuing the service, and as a result, a dedicated elderly medicine unit is currently under construction within our current ED. The activity of the service operating out of this unit will form the next PDSA cycle of this quality improvement project.

\section{Conflict of interest statement}

None declared. 